\title{
HBA1C AND INSULIN RESISTANCE AS PREDICTORS FOR THE SEVERITY OF CORONARY ARTERY DISEASE AND SYSTOLIC HEART FAILURE IN NON DIABETIC PATIENTS.
}

\author{
Mohammad Wafaie abo El-Enin, Ahmed Shafie Ammar, EL Sayed Mohammed Farag and Mohamed \\ Ibrahim Amin* \\ Cardiology Department, Faculty of Medicine, Zagazig University.
}

\begin{abstract}
Background: Coronary heart disease (CAD) is the main cause of morbidity and mortality in developed countries, its prevalence is increasing in developing countries. ${ }^{(1)}$ Insulin resistance (IR) has known to be associated with coronary artery disease (CAD), assessment of severity of the CAD based on IR has not been established in details. ${ }^{(2)}$ Multiple angiographic scoring systems were used systematically to evaluate number of affected epicardial vessels, detailed assessment of lesion severity and quantification of the functional significance of myocardium affected by stenotic lesions. ${ }^{(3)} \mathrm{HbAlc}$ is a useful index of glucose intolerance and hyperglycemia, even when fasting glucose concentrations are normal. ${ }^{(4)}$

Aim of the work: to study the relationship between HbAlc, IR as predictors for the severity of CAD in chronic stable angina and Systolic Heart Failure in Non-Diabetic Patients.

Patient and methods: This study was done in Cardiology department, Zagazig University on 100 patients; 64 males (64\%) \& 36 females (36\%), our patients with CAD were grouped according to Ejection Fraction (EF) into G1 with $\mathrm{EF}>50 \%, \mathrm{G} 2$ with $\mathrm{EF}<50 \%$. They were fatherly divided according to positive family history (FH) of DM; G1a with -ve FH, G1b with +ve FH, G2a with -ve FH, G2b with +ve FH. We searched for IR by measuring HOMA-IR and measured fasting Blood sugar (FBS) and HbA1c as markers of glycaemia. Coronary angiography was done and severity of coronary artery disease was calculated by Gensini score.

Results: In our study we discovered a cutoff point $=5.5 \%$ for $\mathrm{HbA} 1 \mathrm{c}$ to predict the systolic heart failure. It showed a good specificity and fair sensitivity $(73.5 \%, 43.9 \%)$ respectively. There was a highly statistical significant difference between $\mathrm{HbA} 1 \mathrm{c}$ and number of coronary arteries affected $(\mathrm{X}=9.7, \mathrm{p}=0.004)$. As regard $\mathrm{HbA1c}$ and Gensini score, there was a highly statistical significant difference $(X=10.5, \mathrm{p}=0.001)$. We also discovered a cutoff point $=1.9$ for HOMA-IR to predict the systolic heart failure in our study. There was a significant relation between Gensini Score and dyslipidemia $(r=0.337, p=0.001)$, hypertension $(r=$ $0.255, \mathrm{p}=0.011)$ and fasting blood sugar $(\mathrm{r}=0.229, \mathrm{p}=0.022)$. There was also a significant correlation between systolic heart failure and dyslipidemia $(\mathrm{r}=0.315, \mathrm{p}=0.001)$ and hypertension $(\mathrm{r}=0.251, \mathrm{p}=0.012)$ in non-diabetic patients.

Conclusions: We conclude that in non-diabetic patients with CAD, HbA1c above the cutoff point $5.5 \%$ can predict an increasing severity of the coronary artery disease and the number of the affected coronary arteries.

Key words: Coronary artery disease, Insulin resistance, Hba1c, Heart Failure, HOMA-IR.
\end{abstract}

\section{INTRODUCTION}

Clycated hemoglobin (hemoglobin A1c, $\mathrm{HbA}_{1 \mathrm{c}}, \mathrm{A} 1 \mathrm{C}$, or $\mathrm{Hb}_{1 \mathrm{c}}$; sometimes also $\mathrm{HbA} 1 \mathrm{c})$ is a form of hemoglobin that is measured primarily to identify the average plasma glucose concentration over prolonged periods of time. It is formed in a non-enzymatic glycation pathway by hemoglobin's exposure to plasma glucose. Normal levels of glucose produce a normal amount of glycated hemoglobin. As the average amount of plasma glucose increases, the fraction of glycated hemoglobin increases in a predictable way. This serves as a marker for average blood glucose levels over the previous months prior to the measurement. ${ }^{(5)}$

The evolution of IR is unique in precedes the onset of diabetes and remains fairly constant throughout the disease process from the time of diagnosis, even after the conventional treatment for type 2 diabetes mellitus. If significant correlation is established between IR and severity of CAD, it will help us in identifying high risk individuals and we might be able to predict the severity by measure of IR, which is a simple test. (6)

The most commonly used angiographic scoring systems from the literature were ten scores, All scores were systematically evaluated on 3 points: (1) Number of epicardial vessels with associated branches incorporated into the score, with higher weight given to scores with more inclusive criteria. (2) Detailed assessment of lesion severity with higher weight given to scores incorporating varying degrees of lesion stenosis and recognition of accepted criteria for hemodynamic or 
interventional significance (ie, $\geq 50 \%$ ). (3) Quantification of the functional significance of myocardium affected by stenotic lesions. With higher weight given to scores incorporating a greater number of epicardial and associated branch vessels. ${ }^{(3)}$ The echocardiographic assessment of LV systolic function plays a pivotal role in the diagnosis, risk stratification and therapeutic guidance of proven medical and interventional therapy in patients with suspected or known cardiac disease. For example, an accurate assessment of LV function is now recommended as part of the management of acute coronary syndrome (ACS). ${ }^{(7)}$

\section{AIM OF THE WORK}

To study the relationship between $\mathrm{HbA1c}$, insulin resistance as predictors for the severity of coronary artery disease in chronic stable angina and systolic heart failure in non-diabetic patients.

\section{Patients and Methods}

This Study has been carried out in cardiology Department; Zagazig University during the period from (August 2012 to December 2013).

The study has included 100 patients; this study is a retrospective study in order to find out the relationship between $\mathrm{HbA1c} \&$ Insulin resistance as predictors for the severity of stable coronary artery disease and systolic heart failure in nondiabetic patients.

Inclusion Criteria: Sample size of the studied group of patients will be statistically calculated. Patients will be divided in to two groups to evaluate the effect of HbAlc \& insulin resistance on both groups, both groups are non-diabetic and both groups will be subdivided in to two groups according to the family history of diabetes mellitus.

Group 1: Patients with chronic stable angina and good systolic function.

Group1a: Patients with chronic stable angina and good systolic function without family history of diabetes mellitus.

Group1b: Patients with chronic stable angina and good systolic function with family history of diabetes mellitus.

Group 2: Patients with chronic stable angina with impaired systolic function.

Group 2a: Patients with chronic stable angina with impaired systolic function without family history of diabetes mellitus.

Group 2b: Patients with chronic stable angina with impaired systolic function with family history of diabetes mellitus.

Exclusion Criteria: Recent Myocardial infarction (STEMI or NSTEMI) within six months, diabetes mellitus (diabetes mellitus will be diagnosed according to WHO criteria: Symptoms of hyperglycemia (e.g. polyuria, polydipsia, unexplained weight loss, visual blurring, genital thrush, lethargy) and raised venous glucose detected once - fasting $>126 \mathrm{mg} / \mathrm{dl}$ or random $>200 \mathrm{mg} / \mathrm{dl}$ or, $\mathrm{HbA} 1 \mathrm{C} \geq 6.5 \%$.the test should be performed in a laboratory using a method that National Glycohemoglobin Standardization Program (NGSP) certified and standardized to the Diabetes Control and Complications Trial (DCCT) ${ }^{(8)}$,Valvular or congenital heart disease, Renal or hepatic impairment, Primary heart muscle disease, sub-clinical Cushing's syndrome and hypogonadism, Hypothyroidism, Abdominal obesity.

All patients included in the study will be subjected to the following: Full history taking: with special interest to determine the risk factors for coronary artery diseases (CAD) as hypertension, smoking, dyslipidemia and positive family history of ischemic heart disease \& DM, Thorough assessment of presence or absence of symptoms of heart failure. If symptoms are present, patients will be classified according to NYHA classification, Assessment of the severity of coronary artery disease by The Gensini scoring which is a valuable aid to estimate the severity of CAD according to angiographic findings, The severity of the coronary lesions was estimated using the Gensini score; based on the degree of luminal obstruction which may involve either concentric or eccentric lesions. The score for each segment was multiplied by a weighing factor reflecting the importance of that particular coronary segment,

General clinical examination, Local cardiac examination, Resting twelve-leads surface ECG, routine laboratory investigations including: CBC, kidney and liver function tests, fasting and two hours postprandial blood sugar, lipid profile and $\mathrm{HbA1c}$ assay \& measurement of Insulin resistance by HMA-IR (Homeostatic Model Assessmentinsulin Resistance), Echo-Doppler examination will be done for all patients: transthoracic conventional echo Doppler done in all standard views at all accessible windows: The following measures will be obtained: interventricular septum thickness (IVST), left ventricular posterior wall thickness (LVPWT), end diastolic dimension (EDD), end systolic dimension (ESD), aortic root diameter (AO), and left atrial diameter (LA), Left ventricular function assessment by calculating percent fractional shortening (\%FS) and ejection fraction (EF) will be assessed by conventional Mmode from the parasternal window and 2D 
Simpson's method from the apical window, Body Mass Index, Random Blood Sugar.

Coronary Angiography: Coronary angiographic data were collected for all patients as an evidence of Ischemic heart disease.

Laboratory Investigation: Blood Glucose Level: the blood sugar concentration: fasting, $2 \mathrm{hr}$ postprandial and hemoglobin A1C were measured in all patients, Homeostatic Model Assessment (HOMA-IR):-

The approximating equation for insulin resistance, in the early model, used a fasting plasma sample, and was derived by use of the insulin-glucose product, divided by a constant HOMA-IR = Glucose $\mathrm{x}$ Insulin / 405 (Glucose in $\mathrm{mg} / \mathrm{dl}$ )

STATISTICAL ANALYSIS.

Data were then imported into Statistical Package for the Social Sciences (SPSS version 20.0) software for analysis. Quantitative data were expressed as means \pm SD. Differences between means in two parametric groups were compared by Student's t test. non-parametric data by Mann-
Whitney test and multiple non parametric groups by Kruskal-Wallis and parametric by ANOVA, correlation by Pearson's correlation. Sensitivity, specificity, positive predictive value (PPV), negative predictive value (NPV) were used to plot Receiving Operative Carve) ROC curve to get cut off point for different parameters. $\mathrm{P}$ value was set at $<0.05$ for significant results $\&<0.001$ for high significant result.

\section{RESULTS}

Concerning the relation of $\mathrm{HbA} 1 \mathrm{c}$ to systolic heart failure $(\mathrm{EF})$ showed a good specificity and fair sensitivity $(73.5 \%, 43.9 \%)$ respectively. with good positive predictive value (PPV), and fair negative predictive value (NPV) $(76.3 \%$ and 40.3\%) respectively.

We discovered a cutoff point $=5.5 \%$ for $\mathrm{HbA} 1 \mathrm{c}$ to predict the systolic heart failure in our study. There was statistical significant difference between $\mathrm{HbA} 1 \mathrm{c}$ and number of coronary vessels affected in our study population $(\mathrm{X}=9.7, \mathrm{p}$ $=0.004)$.

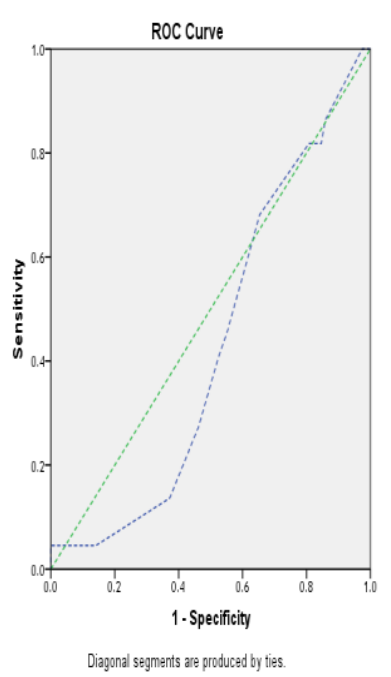

Fig (1): Roc curve shows relation of $\mathrm{HbA} 1 \mathrm{c}$ to systolic heart failure sensitivity and specificity.

As regard the HbA1c and Gensini score for detection of severity of coronary vessel affected there was a highly statistical significant difference in our population study $(X=10.5, p=0.001)$. In our study we also discovered a cutoff point $=1.9 \%$ for HOMA-IR to predict the systolic heart failure in our study. 


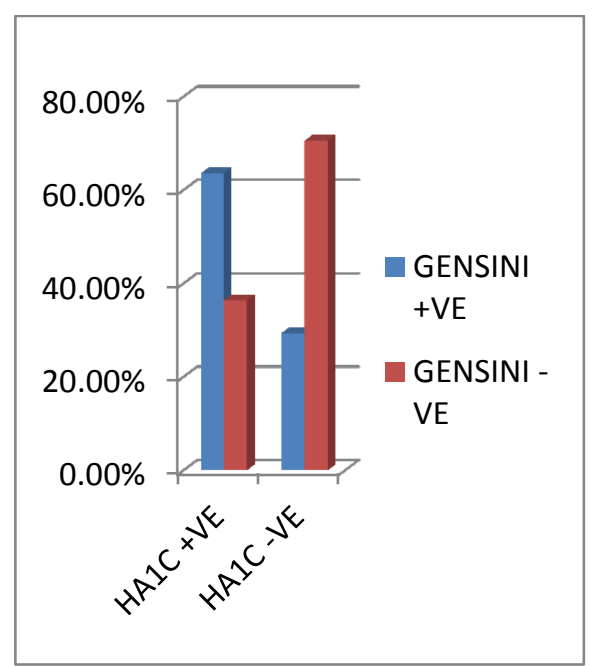

Fig (2) Association between cutoff point to HbA1c \&Gensini score above and below EF $50 \%$ ( Gensini +ve above $50 \%$, -ve below 50\%).

We found the patient over 1.9 had more affected coronary arteries and also had more sever coronary artery disease pattern despite its not reaching the statistically significant difference, There was no statistically difference between HOMA-IR and number of affected coronary vessels and Genseni score in our study population $(\mathrm{X}=3.3, \mathrm{p}=0.4),(\mathrm{X}=0.06, \mathrm{p}=0.8)$ respectively.

Concerning correlations between Gensini score - as a marker of severity of coronary artery disease - versus other variables, there was highly significant positive correlation between Gensini score and dyslipidemia ( $r$ $=0.337, \mathrm{p}=0.001$ ).

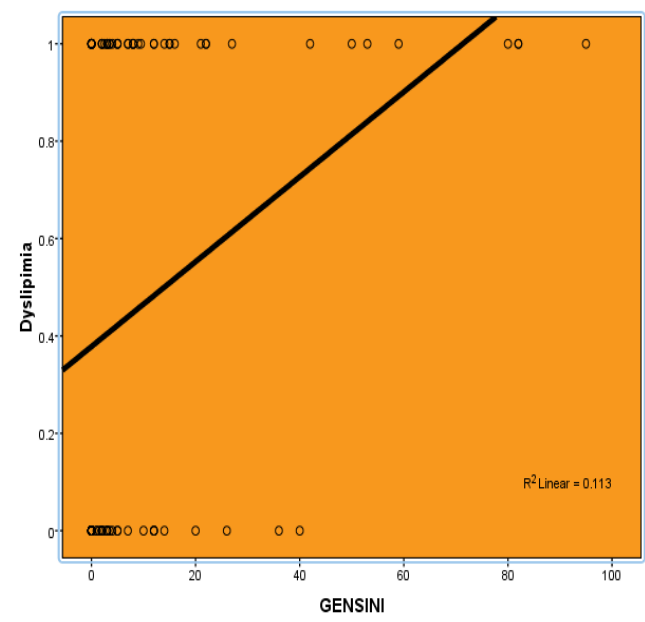

Fig (3): Correlation between Gensini score and dyslipidemia.

Significant positive correlations between Gensini score and both hypertension and FBS $(r=0.255, p=$ $0.011),(r=0.229, \mathrm{p}=0.022)$ respectively.

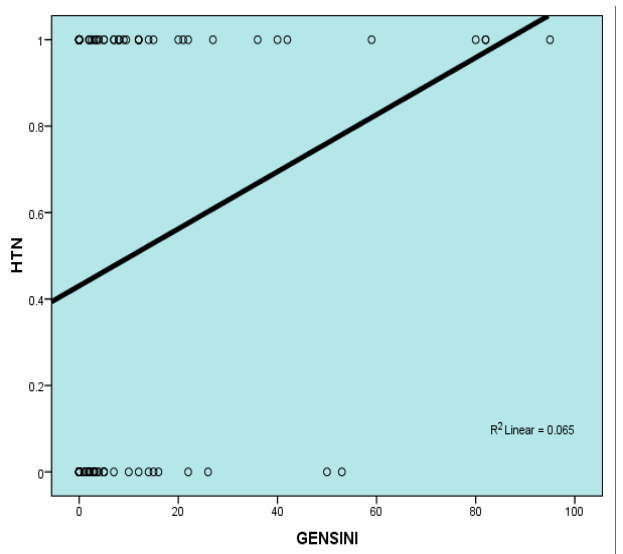

Fig (4): Correlation between Gensini score and Hypertension. 


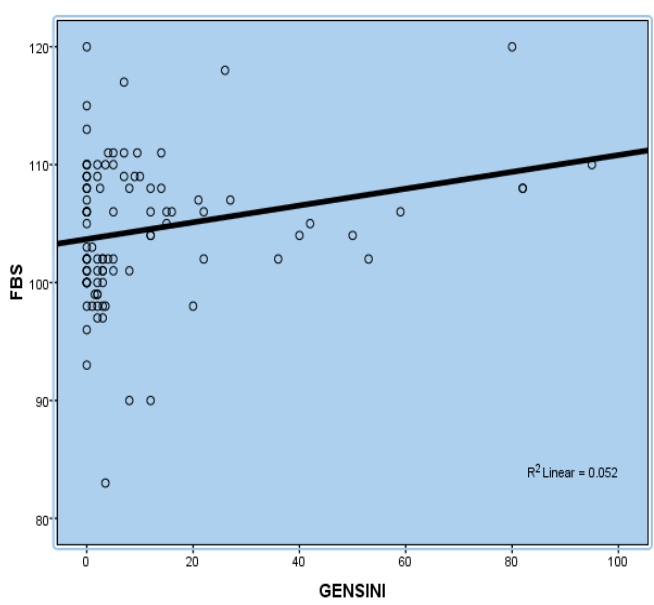

Fig (5): Correlation between Gensini score and fasting blood sugar.

On performing multiple regression analysis to detect predictors of severity of coronary artery disease (Gensini Score), dyslipidemia was proved to be a true predictor $(\mathrm{R}=0.489, \mathrm{p}=0.004)$.

Table (1): shows regression analysis to detect the predictors of Gensini score.

\begin{tabular}{|c|c|c|c|}
\hline & Predictors & $R$ & $" p "$ \\
\hline \multirow{4}{*}{$\begin{array}{l}\text { Gensini } \\
\text { score } \\
\text { Vs }\end{array}$} & Hypertension & \multirow{4}{*}{0.489} & 0.03 \\
\hline & Age & & 0.01 \\
\hline & Dyslipidemia & & 0.004 \\
\hline & $F B S$ & & 0.063 \\
\hline
\end{tabular}

Concerning correlations between ejection fraction measured by modified Simpson method -as a marker of systolic heart failure- versus other variables, there was highly significant positive correlation between ejection fraction and dyslipidemia $(\mathrm{r}=0.315, \mathrm{p}=0.001)$.

Significant positive correlations between ejection fraction and hypertension $(r=0.251, p=0.012)$.

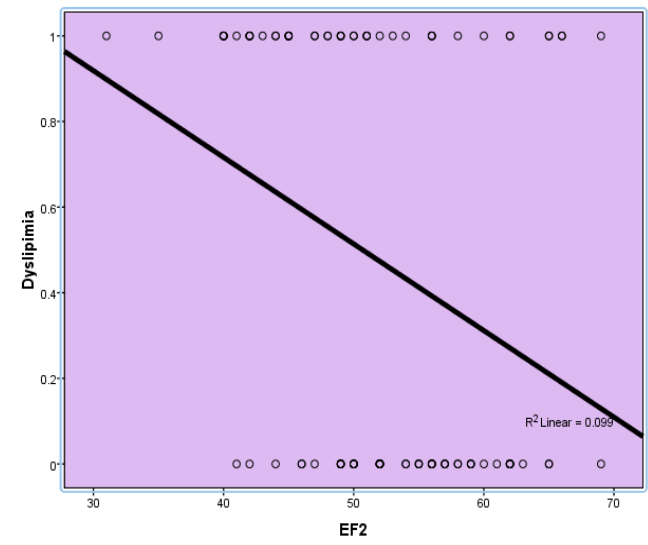

Fig (6): Correlation between Ejection fraction (by simpson method) and dyslipidemia. 


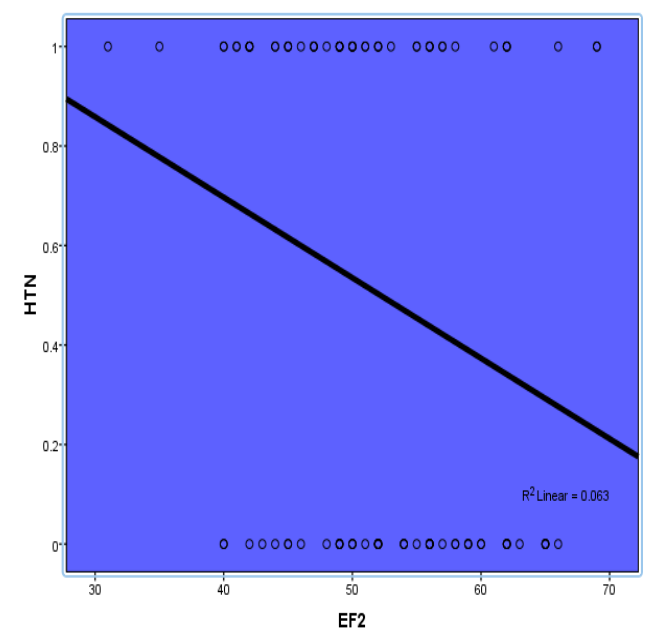

Fig (7): Correlation between Ejection fraction (by simpson method) and Hypertension.

On performing multiple regression analysis to detect predictors of systolic heart failure; dyslipidemia and hypertension were proved to be true predictors for systolic heart failure $(\mathrm{R}=0.489, \mathrm{p}=0.05$ ), for Hypertension $(\mathrm{R}=0.489, \mathrm{p}=0.039)$.

Table (2): shows regression analysis to detect the predictors of systolic heart failure.

Predictors R $\quad$ R

Systolic Heart Failure

Dyslipidemia

Hypertension
0.005

0.372

0.039

\section{DISCUSSION}

Gerstein HC et al, $2003^{(9)}$ evaluated the relation between the severity of coronary atherosclerosis and HbAlc levels in non-diabetic patients. They didn't find any relationship between HbAlc and extent of coronary involvement assessed by Gensini score.

(Ashraf H et al, 2013) ${ }^{(10)}$ Showed that in their study included one hundred forty seven patients with increasing $\mathrm{HbAlc}$ levels, there was a significant increase in the prevalence of CAD and number of vessels involved. In multivariate analysis, $\mathrm{HbA1c}$ emerged as an independent predictor of significant CAD (OR: $2.8,95 \%$ CI: 1.3-6.2, $p=0.009)$. Adjusted ORs for the occurrence of CAD were highest in subjects with both hsCRP and HbA1c in the upper 2 quartiles (OR: $4.183 ; \quad 95 \% \quad$ CI: $1.883-9.290, \quad p<$ 0.0001).There was a significant association between Gensini score and increasing $\mathrm{HbAlc}$ tertiles $(p=0.038)$. The ideal cut-off value of $\mathrm{HbA} 1 \mathrm{c}$ for prediction of the occurrence of CAD was $5.6 \% 38 \mathrm{mmol} / \mathrm{mol}$ ) (sensitivity: $60.5 \%$, specificity: 52\%). They concluded that, in nondiabetic subjects, HbA1c could be utilized for risk stratification of CAD and its severity, independent of traditional cardiovascular risk factors, insulin resistance and inflammatory markers In our study we discovered a cutoff point $=5.5$ for $\mathrm{HbA} 1 \mathrm{c}$ to predict the systolic heart failure. It showed a good specificity and fair sensitivity $(73.5 \%, 43.9 \%)$ respectively. There was statistical significant difference between $\mathrm{HbA} 1 \mathrm{c}$ and number of coronary vessels affected in our study population $(\mathrm{X}=9.7, \mathrm{p}=0.004)$. Regarding the HbA1c and Genseni score for detection of severity of coronary vessel affected there was a highly statistical significant difference in our population study $(\mathrm{X}=10.5, \mathrm{p}=0.001)$.

Srinivasan et al,2014, ${ }^{(2)}$ demonstrated that Insulin resistance (IR) has known to be associated with coronary artery disease (CAD), but the assessment of severity of the CAD based on IR has not been established in detail. Also in our study we find that HOMA-IR has no statistical significant regarding number of coronary vessels affected and Gensini score.

In our study we were recruiting non-diabetic patients with HbA1c below $6.5 \%$ and this was not the case in some studies which demonstrated a significant positive correlation between $\mathrm{HbA} 1 \mathrm{c}$ above $6.5 \%$ and coronary angiographic scores, indicating it as a marker of extensive coronary arterial disease (Khaw et al, 2006) ${ }^{(11)}$. The results 
of our study showed a highly significant positive correlation between Gensini score and dyslipidemia $(\mathrm{r}=0.337, \mathrm{p}=0.001)$, significant positive correlations between Gensini score and both hypertension and FBS $(r=0.255, \mathrm{p}=0.011)$, $(\mathrm{r}=0.229, \mathrm{p}=0.022)$ respectively. Moreover there was significant negative correlation between Gensini score and both $\mathrm{HbA1C}(\mathrm{r}=0110, \mathrm{p}=0$ $.274)$ and HOMA-IR $(\mathrm{r}=0.159, \quad \mathrm{p}=0.115)$ respectively.

Tomova et al, $2012^{(12)}$ demonstrated that, among the non-diabetic patients with heart failure, HbA1C levels did not predict survival outcomes. The retrospective cohort study is published online March 27, 2012 in the American Journal of Cardiology.

As regard the correlations between ejection fraction measured by modified Simpson method as a marker of systolic heart failure- versus other variables, there was highly significant positive correlation between ejection fraction and dyslipidemia ( $\mathrm{r}=0.315, \mathrm{p}=0.001$ ), significant positive correlations between ejection fraction and hypertension $(\mathrm{r}=0.251, \mathrm{p}=0.012)$. Also there was no significant correlation between systolic heart failure and both $\mathrm{HbA1C}(\mathrm{r}=0132, \mathrm{p}=0$.189) and HOMA-IR ( $\mathrm{r}=0.073, \mathrm{p}=0.470)$ respectively.

Conclusions: We conclude that in non-diabetic patients with $\mathrm{CAD}, \mathrm{HbA} 1 \mathrm{c}$ above the cutoff point $5.5 \%$ can predict an increasing severity of the coronary artery disease and the number of the affected coronary arteries.

\section{REFERENCE}

1. Modan M, Meytes D, Rozeman P, Yosef SB, Sehayek E, Yosef NB. Significance of high HbA1c levels in normal glucose tolerance. Diabetes Care 1988; 11:422-8.

2. Srinivasan MP1, Kamath PK2, Manjrekar PA3, Unnikrishnan B4, Ullal A5, Kotekar MF5, Mahabala C1.North American journal of medical sciences, ISSN: 2250-1541,Pages:6114,May 2014.

3. American Heart J. Oct 2012; 164(4): 547552.e1.

4.Tsuji I, Nakamoto K, Hasegawa T, Hisashige A, Inawashiro H, Fukao A. Receiver operating characteristic analysis on fasting plasma glucose, HbA1c, and fructosamine on diabetes screening. Diabetes Care 1991;14:1075-7.

5.Larsen ML, Hørder M, Mogensen EF (1990). "Effect of long-term monitoring of glycosylated haemoglobin levels in insulindependent diabetes mellitus". N. Engl. J. Med. 323 (15): 1021-5.

6. Kim JY, Mun HS, Lee BK, Yoon SB, Choi EY, Min PK. (2010): Impact of metabolic syndrome and its individual components on the presence and severity of angiographic coronary artery disease. Yonsei Med J; 51:676-82.

7. Harvey Feigenbaum, Lippincotts, Williams \& Wilkins (2009): Echocardiography, ed. Feigenbaum, 9th Edition, updated 2013, text book.

8. American Diabetes Association, ADA, 2013.

9.Gerstein HC, Anand S, Yi QL, Vuksan V, Lonn $\mathrm{E}$, Teo $\mathrm{K}$. The relationship between dysglycemia and atherosclerosis in South Asian, Chinese, and European individuals in Canada: a randomly sampled cross-sectional study. Diabetes Care 2003;26:144-9.

10. Ashraf H. , Boroumand M.A., Amirzadegan A., Talesh S.A., and Davoodi G. Hemoglobin A1C in non-diabetic patients: An independent predictor of coronary artery disease and its severity. Diabetology and Metabolic Syndrome. published online 31 October 2013.

11. Khaw KT, Wareham N. Glycated hemoglobin as a marker of cardiovascular risk. Curr Opin Lipidol 2006;17:637-43.

12.Tomova GS, Nimbal V, Horwich TB. Relation between hemoglobin $\mathrm{A}_{1 \mathrm{c}}$ and outcomes in heart failure patients with and without diabetes mellitus. Am J Cardiol 2012. 